\title{
Effect of diamond surface orientation on the Thermal Boundary Conductance between diamond and aluminum
}

\author{
Christian Monachon*,a, Ludger Weber ${ }^{\mathrm{a}}$ \\ ${ }^{a}$ Laboratoire de Métallurgie Mécanique, Ecole Polytechnique Fédérale de Lausanne, \\ Lausanne, Switzerland
}

\begin{abstract}
[100] and [111] oriented diamond substrates were treated using Ar:H and Ar:O plasma treatments, and $1: 1 \mathrm{HNO}_{3}: \mathrm{H}_{2} \mathrm{SO}_{4}$ heated at $200^{\circ} \mathrm{C}$. Subsequent to these treatments, an aluminum layer was either evaporated or sputtered on the substrates. The Thermal Boundary Conductance (TBC) as well as the interfacial acoustical reflection coefficient between this layer and the diamond substrate was then measured using a Time Domain ThermoReflectance (TDTR) experiment. For the Ar:H plasma treated surfaces the [111] oriented faces exhibited conductances $40 \%$ lower than the [100] oriented ones, with the lowest measured TBC at $32 \pm 5 \mathrm{MWm}^{-2} \mathrm{~K}^{-1}$. The treatments that led to oxygen-terminated diamond surfaces (i.e. acid or Ar:O plasma treatments) showed no TBC anisotropy and the highest measured value was $230 \pm 25 \mathrm{MWm}^{-2} \mathrm{~K}^{-1}$ for samples treated with Ar:O plasma with a sputtered Al layer on top. Sputtered layers on oxygen-terminated surfaces showed systematically higher TBC than their evaporated counterparts. The interfacial acoustic reflection coefficient correlated qualitatively with TBC when comparing samples with the same type of surface terminations $(\mathrm{O}$ or $\mathrm{H})$, but this correlation failed when comparing $\mathrm{H}$ and $\mathrm{O}$ terminated interfaces with each other.
\end{abstract}

Key words: Time Domain ThermoReflectance, Thermal Boundary Conductance, Metal Matrix Composites, Aluminum, Diamond

\footnotetext{
*. Corresponding author

Email addresses: christian.monachon@epfl.ch (Christian Monachon), ludger.weber@epfl.ch (Ludger Weber)
} 


\section{Introduction}

Diamond-based Metal-Matrix Composites (MMC) are currently investigated because of their potential as next generation packaging materials for thermal management[1]. Indeed, the high thermal conductivity of diamond and its low coefficient of thermal expansion combined in a composite with that of various metals, should be appropriate for efficient cooling of semiconductor-based devices with significantly reduced levels of differential thermal expansion. So far thermal conductivity as high as $980 \mathrm{Wm}^{-1} \mathrm{~K}^{-1}[2]$ has been achieved in diamond/metal composites.

To maximize the conductivities of such composites, large diamonds (typically $>300 \mu \mathrm{m}$ in size) are used, since the conductance of the diamond/metal interface is finite, and therefore the number of interfaces between metal and diamond matters. This finite thermal conductance of interfaces, termed as Thermal Boundary Conductance (TBC) is thus a critical parameter for decreasing the diamond particle size in metal/diamond composites, which would enable smaller pieces to be produced and improve the machinability of the composites. Particularly in non-reactive systems (e.g. Ag-diamond, Cu-diamond), the TBC is low, leading to much reduced composite conductivities. The TBC can be improved by either adjunction of reactive alloying elements to the matrix, e.g. $[6,7]$ or by modifying the diamond surface[8, 11].

A particularly interesting case is the Al-diamond system, for which a difference in bonding strength has been observed depending on the orientation of the diamond surface[3, 12, 13], the adhesion of the metal on the [100] faces being typically better than on the [111] faces of the typically cuboctahedral synthetic diamond particles used in such composites. The origin of this seemingly better adhesion is not clear. In one case[12], it was concluded that the formation of an interfacial carbide on the diamond [100] face promotes interfacial bonding. In another case[3], still on the [100] face, an oxy-carbide was observed, but was not considered to have a significant impact on the interfacial bonding. The [111] face of the diamond, on the other hand, has been observed not to adhere well to the matrix and be free of reaction products. While concomitance between good adhesion and high TBC is generally observed (e.g. $[4,14,15])$, some groups have pushed the analogy farther by invoking that the $\mathrm{Al} /$ diamond $\mathrm{TBC}$ is anisotropic in such composites, and thus estimated the effect of diamond shape (i.e. proportions of [111] to [100] 
faces) on the composite thermal conductivity $[16,17]$. However, there is no direct experimental evidence for such an anisotropy in Thermal Boundary Conductance for the $\mathrm{Al} /$ diamond couple.

There are only few studies addressing the orientation dependence of TBC, i.e. Hopkins et al.[18] found a strong dependence for $\mathrm{Al}$ on sapphire oriented [0001] or [11 $\overline{2} 0]$ but no difference for $\mathrm{Al}$ on [100] or [111]-oriented Si. Lyeo and Cahill[19] could not find any significant influence of the substrate orientation on TBC for $(\mathrm{Pb}, \mathrm{Bi})$ on $\mathrm{Si}[100]$ or $[111]$ surfaces.

Though no anisotropy has been observed on $\mathrm{Si}$, anisotropy in metal/diamond TBC cannot be excluded a priori. Diamond can have several surface morphologies and terminations, depending whether it is hydrogen[20] or oxygen[21, 22 -terminated, or $\mathrm{sp}^{2}$ reconstructed, and the specific termination again depends on the crystallographic orientation of the surface. The termination of a diamond surface has already been shown to have an effect on the TBC between diamond and metals[19, 23, 24], as well as other interfacial properties[25-28]. Since the reconstruction of a diamond surface depends both on its orientation and its termination[20,21] diamond has the potential to show an orientation-dependence of its TBC, and adhesion, with metals. The present work uses a Time Domain ThermoReflectance (TDTR) experiment to detect a possible orientation dependence of the TBC between aluminum and diamond. In parallel, an estimation of the interfacial stiffness, which is linked to adhesion, in the same system is also made by measuring the interfacial acoustical reflection coefficient by acoustic echoes using the same experimental system.

\section{Experimental}

\subsection{Sample preparation}

Diamonds were purchased from Element 6 (Shannon Airport, Shannon, Co. Clare, Ireland, MWS L25 for [100]-oriented stones, MD111/11 for [111]oriented stones). The [100]-oriented stones were factory-polished, but the [111]-oriented ones were mounted on a sample holder and polished on cast iron for about 3 hours using olive oil and diamond suspensions of 15, 6 and $1 \mu \mathrm{m}$ in size to have a smooth surface finish. All the diamonds were then polished on a regular polishing lap using diamond suspensions of 6 , followed by $1 \mu \mathrm{m}$ size. RMS roughness was checked to be less than $1.5 \mathrm{~nm}$ by FIB cross-section in the areas of interest for the measurement. After polishing, the samples were rinsed with acetone, ethanol and finally isopropanol. This 
state will later be referred to as "As Received" (AR). The surface condition of the diamonds was then modified using 3 different treatments.

\subsubsection{Hydrogen plasma treatment}

A hydrogen plasma treatment was conducted in a Balzers BAI730D chamber, using a 95:5 Ar:H mixture at a pressure of $10^{-3}$ mbar. The plasma was produced between an ionization chamber based on a hot tungsten filament at the top of the reactor and a copper cone at its bottom, using a DC current of $80 \mathrm{~A}$. The plasma had a columnar shape producing a radial diffusive flux of ions and radicals used for the treatment. The recombination enthalpy of the atomic $\mathrm{H}$ was used to heat up our samples. The temperature was monitored using thermocouples protected with zirconia pieces inserted next to the samples. The obtained temperatures are only approximative since the zirconia surface reactivity is probably not the same as that of diamond, but we are confident that the indicated temperatures are within $50{ }^{\circ} \mathrm{C}$ of the actual value for our diamonds. The diamonds were put on Ta sample holders coated with boron nitride to prevent the formation of tantalum carbide. 2 diamonds were put at 13 and $16 \mathrm{~cm}$ from the center of the plasma, reaching respective temperatures of 900 and $700{ }^{\circ} \mathrm{C}$. The treatment conditions were maintained for 2 hours to make sure the whole surface had reacted.

\subsubsection{Acid treatment}

Samples were put in a boiling solution of $1: 1$ (by volume) $\mathrm{HNO}_{3}: \mathrm{H}_{2} \mathrm{SO}_{4}$ (63\% : $98 \%$ ) heated at $200^{\circ} \mathrm{C}$ for $10 \mathrm{~min}$. Though the exact composition of the bath may vary, this type of procedure is known to produce on oxidized surface[20, 29, 30].

\subsubsection{Oxygen plasma treatment}

Samples were treated in a Fischione model 1020 plasma cleaner. The gas composition of the plasma is $\mathrm{Ar}_{2} \mathrm{O}_{2}$ 3:1. The average energy of the atoms is $13 \mathrm{eV}$, with a high yield of oxygen dissociation in order to enhance the chemical reactivity with the surface.

XPS results on these 3 treatments (reported elsewhere [24]) suggest that the produced surfaces are respectively hydrogenized for the first treatment and AR state, and oxidized for the latter two states, as expected.

\subsubsection{Layer deposition}

Samples of Al layers on diamond of about $100 \mathrm{~nm}$ were prepared by evaporation in an Alcatel EVA 600 e-beam evaporator and by sputtering in a 
Balzers BAS 450 sputter deposition system. The deposition speeds used were of $14 \AA_{s^{-1}}$ for the evaporated layers, measured by a quartz microbalance and verified by SEM afterwards, and $6 \AA_{s^{-1}}$ for the sputtered layers, measured by recording the deposition time and measuring the layer thickness by SEM afterwards. For the subsequent verifications by SEM, a piece of passivated $\mathrm{Si}$ wafer was put in the deposition machine at the same time as the diamonds for each deposition, which in our experience yields layers of the same thickness as the ones deposited on diamond.

\subsection{Time Domain ThermoReflectance}

\subsubsection{Experimental setup}

The experimental setup used for the following experiments is a coaxial two tints pump/probe experiment[31] and has already been described in detail[15]. In a nutshell, this setup uses a Spectra Physics Tsunami femtosecond laser working at $80 \mathrm{MHz}$ repetition rate, the beam of which is split into two parts, one used to heat up the sample surface (the pump), and one to test the reflectivity of the sample surface (the probe). The pump beam passes through a mechanical delay stage and the probe beam follows a path of about the same length. Both beams are focused on the same spot on the sample's surface. Varying the length of the optical path of the pump part allows for the creation of a delay between the arrival of the pump and probe on the sample surface from -0.03 to $4.02 \mathrm{~ns}$. The pump beam is also modulated with an electro-optic modulator at a frequency of $10.7 \mathrm{MHz}$ to enable lock-in amplification of the resulting signal. The probe signal is monitored using a $1 \mathrm{~ns}$ rise time photodiode. The signal is passed through a frequency filter centered around $10.7 \mathrm{MHz}$ and then fed into a pre-amplifier before lock-in detection. The X/Y ratio of the values measured by the lock-in was calculated rather than just the X-signal, because it decreases the impact of a change in the overlap of the pump and probe spots[32], which can be a problem when using a mechanical delay stage and a small pump spot size. The size of both the spots was measured using a CMOS camera at 0 and $4 \mathrm{~ns}$ in order to take into account a change in spot size over the course of the delay stage. A $\mathbf{1 0 X}$ objective was used to focus the beam, achieving spots of about 5 $\mu \mathrm{m} e^{-2}$ radius. This lead to fluences of about $0.3 \mathrm{mJcm}{ }^{-2}$, which means that the sample surface is heated up by a few $K$ at most. A background signal was recorded after each measurement, with the probe beam cut, to extract any influence of light directly coming from the pump beam or of electronic noise. 


\subsubsection{TBC measurement}

For the measurement of TBC datasets covering delay times from -0.03 to $4.02 \mathrm{~ns}$ were recorded. To extract the values of TBC the obtained experimental data were fitted using the model first proposed by Cahill[32, 33]. The main fitting parameter is the thermal boundary conductance, though the diamond substrate conductivity had to be allowed to vary between 1000 and $1600 \mathrm{Wm}^{-1} \mathrm{~K}^{-1}$ close to room temperature, which is not an unusual range for nitrogen containing synthetic diamonds $[6,11]$. For a given diamond substrate the best-fit conductivity stayed consistently within $10 \%$ of its mean value. Apart from variations in nitrogen content, the apparent conductivity of the substrate is also affected by spot size and spot shape due to ballistic phonon effects $[24,34]$.

\subsubsection{Interfacial stiffness measurement}

Interfacial stiffness calculations were performed only on treated samples. For this measurement datasets were used covering delay times up to 300 ps and using a smaller step size to capture the acoustic echos of the strain pulse induced by the initial heating of the surface[35]. The background composed of electronic and phononic temperature informations was extracted using a double exponential curve, to isolate each pulse and measure its amplitude by taking the top of an echo and measuring the lowest point within the previous 5 ps. The obtained amplitude data (generally on the first 5 pulses) were then fitted with a simple exponential, extracting a damping rate of the acoustic wave at each reflection. The theoretical reflection coefficient $|r|$ of the interface was calculated as follows :

$$
|r|^{2}=\frac{\left(Z_{A l}-Z_{C}\right)^{2}}{\left(Z_{A l}+Z_{C}\right)^{2}}
$$

with $Z$ the acoustic impedances of the materials $\left(Z_{i}=\rho_{i} v_{i}\right.$ with $\rho$ the density and $v$ the longitudinal sound velocity of the material $i$ ).

\section{Results}

Figure 1 shows the typical range of measurements performed in this study. We do not present AR data even though some data exhibited even lower conductances, because a previous study has found that the result this type of substrate yields are highly variable[15]. The presented data go from 30 to $250 \mathrm{Wm}^{-2} \mathrm{~K}^{-1}$, respectively the lowest and highest TBC obtained on our 


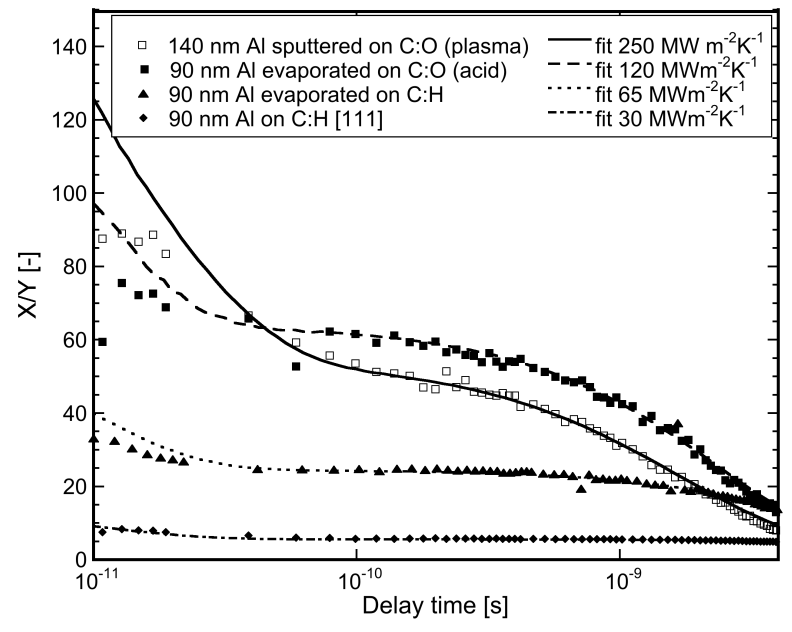

FiguRE 1: Examples of raw TDTR data, along with their corresponding fits for four different samples. The squares show a cooling curve obtained from a [100] diamond treated with an Ar:O plasma with $140 \mathrm{~nm}$ Al sputtered on top. The filled squares are a [100] substrate oxidized using a mixture of $1: 1 \mathrm{HNO}_{3}: \mathrm{H}_{2} \mathrm{SO}_{4}$ heated to $200^{\circ} \mathrm{C}$ with a $90 \mathrm{~nm} \mathrm{Al}$ layer evaporated on top. The filled triangles are a [100] substrate, treated with an Ar:H plasma at $900^{\circ} \mathrm{C}$, with a $90 \mathrm{~nm} \mathrm{Al}$ layer deposited on top. Finally, the filled diamonds represent a [111] substrate treated with an $\mathrm{Ar}: \mathrm{H}$ plasma at $700^{\circ} \mathrm{C}$ with a $90 \mathrm{~nm} \mathrm{Al}$ layer evaporated on top. The thermal properties used in the model were the following ( $\kappa$ is the conductivity, $\mathrm{C}$ the volumetric heat capacity) : $\kappa_{A l}=237 \mathrm{Wm}^{-1} \mathrm{~K}^{-1}, \mathrm{C}_{A l}=2440000$ $\mathrm{Jm}^{-3} \mathrm{~K}^{-1}, \mathrm{C}_{\text {diamond }}=1840000 \mathrm{Jm}^{-3} \mathrm{~K}^{-1}$.

treated samples. Figure 2 shows examples of treatment of the acoustic echoes obtained in the short delay measurements. The samples presented cover the whole range of values measured in this study. Figure 3 shows the TBC results obtained for all the surface treatments and deposition methods described in the experimental section, over 4 measurements, each at different places on the prepared sample. Figure 4 shows the acoustic reflection coefficients $|r|$ measured as a function of the orientation, surface treatment, deposition technique and measured TBC of all our plasma-treated samples.

\section{Discussion}

In Figure 1, conductivity data for bulk $\mathrm{Al}$ are used for data extraction even though the thermal conductivity of a thin film is expected to be lower than that of the bulk[36]. Indeed, for the layers 


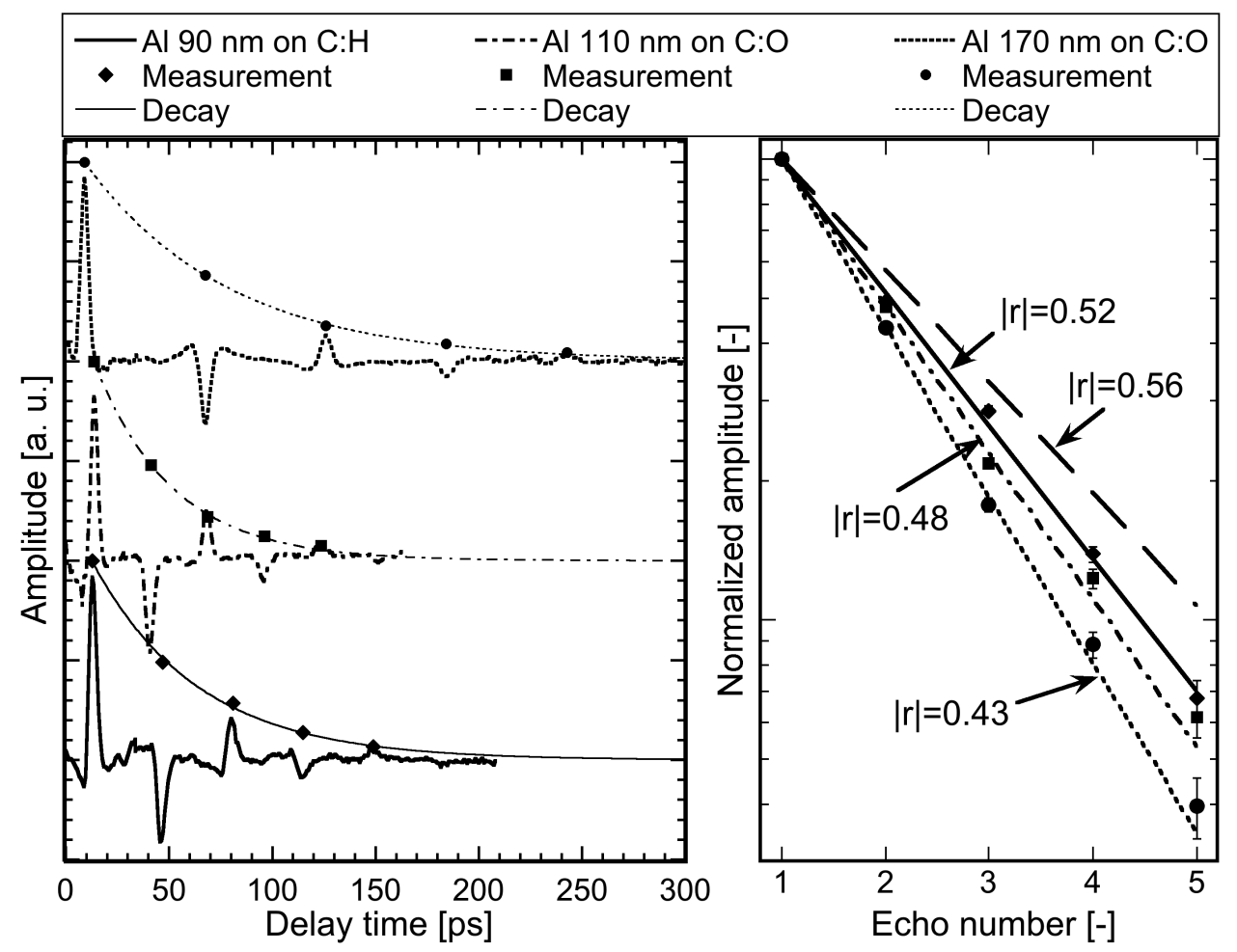

FigURE 2: a) Example of the treatment procedure used to calculate the interface reflection coefficient $|r|$ of longitudinal acoustic waves produced by rapid heating of the metal surface. The filled circles are for a $170 \mathrm{~nm}$ layer of $\mathrm{Al}$ sputtered on an Ar:O plasma treated diamond surface, filled squares are for the same surface with a $90 \mathrm{~nm}$ evaporated $\mathrm{Al}$ overlayer. Filled diamonds are for a $110 \mathrm{~nm} \mathrm{Al} \mathrm{layer} \mathrm{treated} \mathrm{with} \mathrm{an} \mathrm{Ar}: \mathrm{H}$ plasma at $700^{\circ} \mathrm{C}$. The first sample has a [100]-oriented surface, whereas the next two samples are [111]-oriented. b) log-linear plot of the obtained fits, indicating the increase in the slope of the obtained fit as $|r|$ decreases, denoting the effects of strong interfacial bonding. The long-dashed lines at $|r|=0.56$ show the theoretical value of the slope according to equation 1 . The parameters used, the density $\rho$ of the materials and their longitudinal speed of sound, $v$ were $\rho_{A l}=$ $2700 \mathrm{~kg} \mathrm{~m}^{-3}, \rho_{\text {diamond }}=3510 \mathrm{~kg} \mathrm{~m}^{-3}, v_{A l}=6250 \mathrm{~m} \mathrm{~s}^{-1}$ and $v_{\text {diamond }}=17500 \mathrm{~m} \mathrm{~s}^{-1}$.

of interest, using a mean free path in $\mathrm{Al}$ of $16 \mathrm{~nm}[37$, the ratio of thickness over mean free path is of 5.6 at least. Using the table proposed by Sondheimer [38] for a ratio of 5 , the expected reduction in thermal conductivity is of $\mathbf{8 \%}$. The lower estimate in Ref. [36] suggests a reduction of $30 \%$ in the worst case. On the other hand, the sensitivity of the model used to fit the data (as described e.g. 


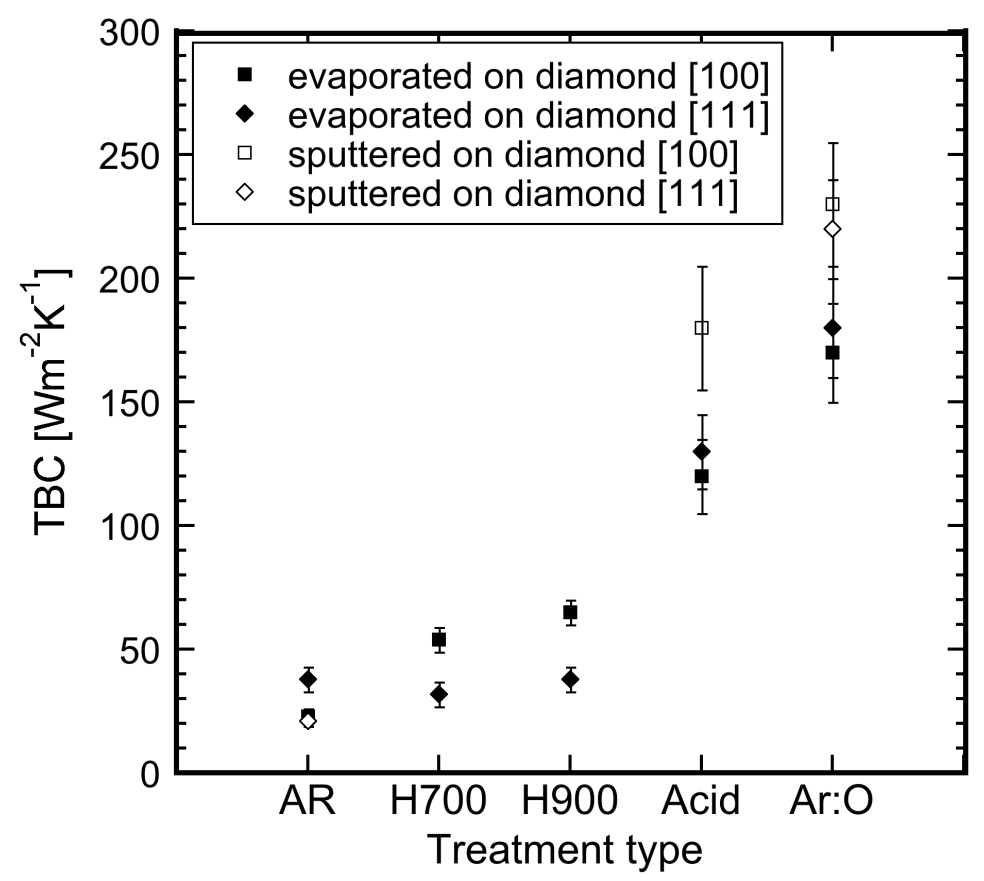

Figure 3: TBC values obtained as a function of the diamond surface treatment applied prior to deposition - respectively from left to right : as received, treated in Ar:H plasma at $700^{\circ} \mathrm{C}$, treated in Ar: $\mathrm{H}$ plasma at $900^{\circ} \mathrm{C}$, treated in acid, treated in Ar:O plasma. The diamonds are for [111]-oriented surfaces and the squares for [100]. Open symbols are for sputtered Al layers, filled symbols for evaporated Al layers. The data on [100] orientation are the same as in Ref. [24].

by Costescu et al[32]) to the layer's conductivity is very low, even for layer conductivities $60 \%$ lower than the bulk value. Hence it does not have a significant impact on both the curves shown and the results extracted from experimental data.

\subsection{Thermal Boundary Conductance}

The TBCs measured for the two surface orientations in Figure 3 are similar within the experimental error for a given oxidizing treatment and $\mathrm{Al}$ deposition technique. For the as-received diamond, only one sample, the [111]-oriented with an evaporated Al layer on top, exhibits a slightly higher TBC as compared to the others. This is explained by the fact that the surface state of these samples is far less controlled as compared to the others and has 


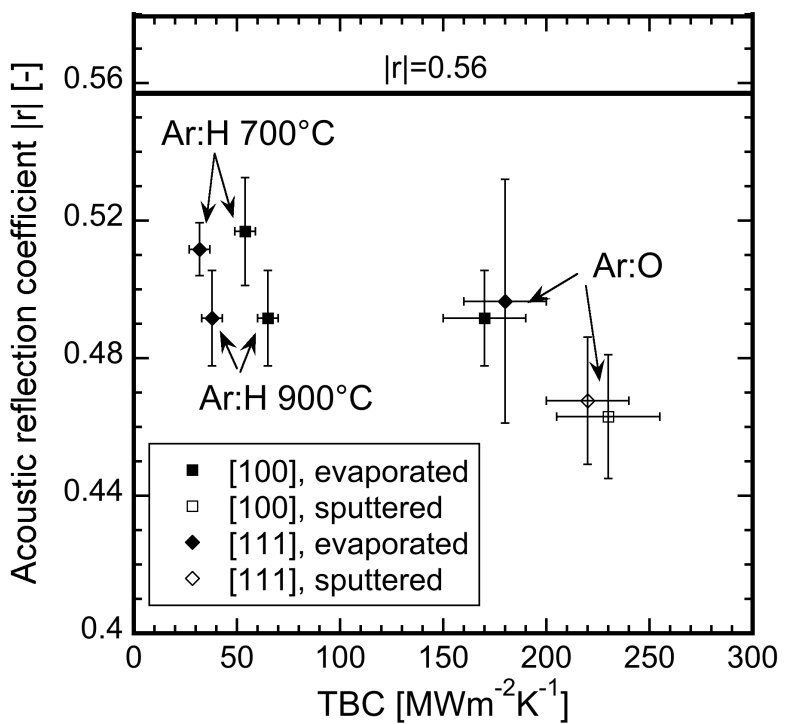

Figure 4: Acoustic reflection coefficients $|r|$, plotted against the TBC measured on the corresponding sample. The open symbols are for sputtered Al layers and the filled ones for evaporated Al layers. Squares are for [100]-oriented samples and diamonds for [111]oriented ones. A line at $|r|=0.56$ is drawn to show where the theoretical limit lies.

already been shown to induce significant differences in measured TBCs[15]. The only significant difference observed is between the hydrogenized [100] and [111] surfaces, the latter exhibiting a $40 \%$ lower TBC as compared to the former in both treatment temperatures applied.

A priori, two effects could be invoked to rationalize the observed effect : surface roughness or surface atomistic structure. An increase in surface roughness has been shown to decrease the conductance of an $\mathrm{Al} / \mathrm{Si}$ interface[39] by as much as $50 \%$ with a roughness passing from 0 to $10 \mathrm{~nm}$. FIB crosssections in the parts investigated by TDTR suggest that the roughness in our case is maintained below $1.5 \mathrm{~nm}$ and a hydrogen plasma treatment has been shown to make the surface smoother rather than rougher in both surface types[40-42]. Besides, a roughness difference would probably have an effect on the TBC measured on oxidized samples as well. It is thus considered that this difference in roughness is unlikely to be the reason for this difference in TBC. The second possible reason is the atomistic structure of the hydrogenized diamond surface. Indeed, the equilibrium structure of a 
[100] surface exposed to hydrogen is $2 \mathrm{x} 1: \mathrm{H}$ with $4 \mathrm{H}$ atoms on 2 classic [100] cubic unit cell $[20,43,44]$, while the $\mathbf{1 \times 1} \mathbf{1} \mathbf{2 H}$ unit cell is unstable due to mutual steric repulsion between the hydrogen atoms. The stable structure of the [111] surface is the $1 \times 1: \mathrm{H}$ and can be terminated either by C-H groups $[20,44]$ or by $\mathrm{C}^{-} \mathrm{H}_{3}$ methyl groups $[45,46]$, increasing substantially the hydrogen surface density. Using the conservative assumption that the [111] $1 \mathrm{x} 1: \mathrm{H}$ surface is only $\mathrm{C}-\mathrm{H}$ terminated, the potential hydrogen adsorption on it is higher by a factor $2 / \sqrt{3}, i$. e. a difference of $15 \%$. Hydrogen termination has both been calculated[47] and measured[27] to be detrimental to $\mathrm{Al} /$ diamond adhesion and therefore interfacial stiffness. Thus higher hydrogen coverage of the plasma-treated [111] face as compared to its [100] counterpart may account for the difference in TBC between the $\mathrm{Al}$ layer and the diamond substrates.

This claim is not confirmed by the measurement of acoustic reflection coefficients shown in Figure 4. The oxidized samples - whether made using an acid mixture or an Ar:O plasma treatment - show no significant orientation dependence but a substantial increase in TBC as compared to both the AR and $\mathrm{Ar}: \mathrm{H}$ treated samples. As compared to $\mathrm{AR}$ values, it increases by a factor of 4.8 if the sample is treated with acid ( 7 if the $\mathrm{Al}$ layer is sputtered) and 7 if the sample is treated with an Ar:O plasma (8.8 if the Al layer is sputtered). This effect has already been observed $[23,24]$, but whether it originates simply from an increased surface reactivity with the metal, from the positive electron affinity that the treatment confers to the surface[27, 41], or from interfacial phononic states [48] remains to be investigated.

\subsection{Interface acoustical reflectivity measurements}

The interface reflection coefficients given in Figure 4 show no significant difference in acoustical reflectivity for equivalent treatment of both surface orientations, though the treatment temperature does seem to have an effect on the Ar:H treated samples. The reflection coefficients obtained are lower than the theoretical value, meaning that there might be other damping phenomena for the acoustical wave produced by the laser heat than transmission to the diamond substrate. We attribute this to dispersion of the wave within the layer and to interface roughness, which has been measured by FIB cross section to be lower than $1.5 \mathrm{~nm}$ on all samples. This prevents us from estimating the stiffness of the observed interfaces since equation 1 implies a reflection coefficient higher than theoretical values.

Experimentally, for each surface orientation and specific surface treatment 
(Ar:H and Ar:O), a lower $|r|$ coincides with higher TBC. The highest TBC and lowest acoustical reflection coefficient were measured in the case of Ar:O plasma treated, sputtered samples. This was expected since sputtered layers are known to adhere better, thereby reducing $|r|$ compared with evaporated layers. This however does not remain true if we consider all the surface treatments together since the Ar:O treated surfaces with $\mathrm{Al}$ evaporated on them show interfaces with the same reflection coefficient as the ones Ar:H-treated at $900^{\circ} \mathrm{C}$. This could be due to the fact that this technique only measures the reflection coefficient of the interface using longitudinal phonons as a probe, and takes no account of shear modes of the interface. Indeed, the latter type of phonon is thought to contribute the most to the TBC. In the main models for TBC available in the literature, especially ones based on the Debye approximation of solids, such as the Acoustic[51] (AMM) and Diffuse[52] (DMM) Mismatch Models and the radiation limit, the phonon flux is proportional to the Debye density of states. This varies as $v^{-3}(v$ is the sound velocity of a specific phonon mode). Therefore, transverse phonons, of lower speed, might contribute more to raise TBC. The transverse acoustical reflection coefficient of the interface is however not probed by the technique used, which may explain why the acoustical reflection coefficient measurements do not correlate perfectly with TBC.

An alternative explanation could simply reside in the fact that only phonons with wavelengths on the order of the layer thickness are present in the wave packet that is sent against the interface, whereas contributions to TBC come from a much wider variety of wavelengths. At this elevated temperature, short wavelength phonon states are highly populated and should contribute much more to thermal transport than the long-wavelength ones probed using picosecond acoustics. A difference in transfer coefficients between the former and the latter would affect TBC and not picosecond acoustics measurements, which could also explain the observed discrepancy.

Finally, it is to be noted that if surfaces are differently populated (e.g. the [111] with $\mathrm{H}$ and [100] with O), the biggest contrast in TBC that could occur would be between the $32 \pm 5 \mathrm{MWm}^{-2} \mathrm{~K}^{-1}$ of [111] oriented surface treated with hydrogen at $700{ }^{\circ} \mathrm{C}$ and the $170 \pm 20 \mathrm{MWm}^{-2} \mathrm{~K}^{-1}$ observed on the $\mathrm{Ar}$ :O treated [100] surface. However, such a situation is unexpected to occur in a MMC since the diamond powders are likely to be randomly terminated and their surface contaminated, therefore corresponding more to the AR state. 
Calculating the average of all the values obtained for the AR state in figure 3 , we obtain a TBC of $28 \pm 8 \mathrm{MWm}^{-2} \mathrm{~K}^{-1}$. Particulate diamondaluminum composites produced with variously sized powders infiltrated with aluminum and analyzed using the approach described in [53] allowed extracting a $\mathrm{TBC}$ of $33 \pm 3 \mathrm{MWm}^{-2} \mathrm{~K}^{-1}$, confirming this hypothesis.

\section{Conclusion}

The Thermal Boundary Conductance between $\mathrm{Al}$ and diamond was investigated varying i) the surface treatments, using Ar:H and Ar:O plasma and boiling in an acid mixture, ii) the surface orientation, using [100] and [111] oriented single crystals, and iii) the Al deposition technique, using evaporation or sputtering. The samples were compared using two techniques based on a Time Domain ThermoReflectance experiment. The first was used to measure the TBC itself and the second to estimate the acoustical reflection coefficient between $\mathrm{Al}$ and diamond using dampening of acoustic echoes. The results can be summarized as follows :

- No significant difference in TBC was observed between the differently oriented substrates produced using the same surface treatments and deposition technique in all cases except the $\mathrm{Ar}: \mathrm{H}$ plasma-treated samples.

- In the case of Ar:H plasma treated samples, an orientation dependence of the $\mathrm{Al}$ /diamond TBC is observed. This is assumed to be due to an increased hydrogen coverage in the case of the [111] oriented sample as compared to [100]. Even then, the greatest difference in TBC between two specimens is that between $54 \pm 5$ and $32 \pm 5 \mathrm{MWm}^{-2} \mathrm{~K}-1$, or $40 \%$.

- The only way a substantial difference could arise in the TBC between $\mathrm{Al}$ and various diamond surface orientations would be if they are differently terminated, or if the production process of the MMC allows for the faces to react differently. As discussed, the first is unlikely to happen since diamond powders are likely to be contaminated in various ways and their surface termination are not as well defined as the ones obtained in this study.

- No difference was observed in interfacial acoustical reflection coefficient between differently oriented substrates in all cases of interest in this study.

- Our measurements suggest that acoustical reflection coefficient values are not directly correlated to $\mathrm{TBC}$, though with equivalent treatment, 
decreasing the acoustical reflection coefficient by changing the treatment temperature or the $\mathrm{Al}$ deposition method increases the TBC.

- The best TBC obtained on evaporated samples was $170 \pm 20 \mathrm{MWm}^{-2} \mathrm{~K}^{-1}$ both on [100] and [111] oriented substrates having undergone an Ar:O plasma treatment, compared to a value of about 30 obtained in composites. Substantial improvements are thus possible in the TBC and hence performance of $\mathrm{Al}$ matrix diamond particles composites for heat management.

- The best TBC between $\mathrm{Al}$ and diamond obtained in this study was $230 \pm 25 \mathrm{MWm}^{-2} \mathrm{~K}^{-1}$ on a [100]-oriented surface treated using an Ar :O plasma, using a sputtered Al overlayer.

\section{Acknowledgments}

Financial support of C. Monachon by the SNSF Project No. 200021121881 and 200020-135132 is gratefully acknowledged. The authors kindly acknowledge Christof Hollenstein and Loïc Curchod for their time and advice as well as the Ar:H plasma treatments. Finally, Professor Hubert Girault of the Laboratoire d'Électrochimie Physique et Analytique (LEPA) at EPFL is acknowledged for providing the laser source for the experiments. Prof David G. Cahill of the University of Illinois at Urbana Champaign is gratefully acknowledged for his helpful pieces of advice.

\section{Références}

[1] C. Zweben, J. Adv. Mater. 39 (2007) 3-10.

[2] R. Tavangar, L. Weber, Merg. Mater. Res. 1, 2 (2012) 67-74.

[3] O. Beffort, F. A. Khalid, L. Weber, P. Ruch, U. E. Klotz, S. Meier, S. Kleiner, Diam. Relat. Mater. 15 (2006) 1250-1260.

[4] L. Weber, R. Tavangar, Scripta Mater. 57 (2007) 988-991.

[5] T. Schubert, L. Ciupinski, W. Zielinski, A. Michalski, T. Weissgärber, B. Kieback, Scripta Mater. 58 (2008) 263-266.

[6] R. Tavangar, J. M. Molina, and L. Weber, Scripta Mater. 56 (2007) 357-360. 
[7] T. Schubert, B. Trinidade, T. Weissgärber, B. Kieback, Mat. Sci. Eng. A 475 (2008) 39-44.

[8] J. Hell, M. Horkel, E. Neubauer, C. Eisenmenger-Sittner, Vacuum 84 (2010) 453-457.

[9] C. Edtmaier, L. Weber, R. Tavangar, Adv. Mater. Res. 59 (2009) $125-130$.

[10] A. M. Abyzov, S. V. Kidalov, F. M. Shakhov, J. Mater. Sci. 46 (2011) 1424-1438.

[11] J.-F. Silvain, A. Veillere, J.-M. Heintz, C. Vincent, T. Guillemet, G. Lacombe, Y. Lu, N. Chandra, Emerg. Mater. Res. 1 (2012) 75-87.

[12] P. W. Ruch, O. Beffort, S. Kleiner, L. Weber, P. J. Uggowitzer, Compos. Sci. Technol. 66 (2006) 2677-2685.

[13] S. Kleiner, F. A. Khalid, P. W. Ruch, S. Meier, O. Beffort, Scripta Mater. 55 (2006) 291-294.

[14] M. D. Losego, M. E. Grady, N. R. Sottos, D. G. Cahill, P. V. Braun, Nat. Mater. Lett. 11 (2012) 502-505.

[15] C. Monachon, L. Weber, Emerg. Mater. Res. 1 (2012) 89-98.

[16] K. Chu, C. Jia, X. Liang, C. Hui, W. Gao, H. Guo, Mater. Des. 30 (2009) 4311-4316.

[17] J. Flaquer, A. Ríos, A. Martin-Meizoso, S. Nogales, H. J. Böhm, Comp. Mater. Sci. 41 (2007) 156-163.

[18] P. E. Hopkins, T. E. Beechem, J. C. Duda, K. Hattar, J. F. Ihlefeld, M. A. Rodriguez, E. S. Piekos, Phys. Rev. B 84 (2011) 1-7.

[19] H.-K. Lyeo, D. G. Cahill, Phys. Rev. B 73 (2006) 144301 1-6.

[20] H. Kawarada, Surf. Sci. Rep. 26 (1996) 205-259.

[21] T. E. Derry, N. W. Makau, C. Stampfl, J. Phys.-Condens. Mat. 22 (2010) $1-12$. 
[22] J. C. Zheng, X. N. Xie, A. T. S. Wee, K. P. Loh, Diam. Relat. Mater. 10 (2001) 500-505.

[23] K. C. Collins, S. Chen, G. Chen, Appl. Phys. Lett. 97 (2010) 1-3.

[24] C. Monachon, L. Weber, J. Appl. Phys. 113 (2013) 183504 1-8.

[25] T. Tachibana, J. T. Glass, R. J. Nemanich, J. Appl. Phys. 73 (1993) 835-842.

[26] H. Kageshima, M. Kasu, Jap. J. Appl. Phys. 48 (2009) 1-5.

[27] F. Maier, J. Ristein, L. Ley, Phys. Rev. B 64 (2001) 1-7.

[28] Y. Mori, H. Kawarada, A. Hiraki, Appl. Phys. Lett. 58 (1991) 940-941.

[29] P. W. May, J. C. Stone, M. N. R. Ashfold, K. R. Hallam, W. N. Wang, N. A. Fox, Diam. Relat. Mater. 7 (1998) 671-676.

[30] S. Kumaragurubaran, T. Yamada, S. Shikata, Diam. Relat. Mater. 17 (2008) 472-475.

[31] K. Kang, Y. K. Koh, C. Chiritescu, X. Zheng, D. G. Cahill, Rev. Sci. Instr. 79 (2008) 1-4.

[32] R. M. Costescu, M. D. Wall, D. G. Cahill, Phys. Rev. B 67 (2003) 1-5.

[33] D. G. Cahill, Rev. Sci. Instr. 75 (2004) 5119-5122.

[34] A. J. Minnich, J. A. Johnson, A. J. Schmidt, K. Esfarjani, M. S. Dresselhaus, K. A. Nelson, G. Chen, Phys. Rev. Lett. 107 (2011) 1-4.

[35] C. Thomsen, H. T. Grahn, H. J. Maris, J. Tauc, Phys. Rev. B 34 (1986) 4129-4138.

[36] G. Chen, P. Hui, Appl. Phys. Lett. 74 (1999) 2942-2944.

[37] S. Kumar, G. C. Vradis, J. Heat Transf. 116 (1994), 28-34.

[38] E. H. Sondheimer, Adv. Phys. 50 (2001), 499-537.

[39] J. C. Duda, P. E. Hopkins, Appl. Phys. Lett. 100 (2012), 111602, 1-3. 
[40] O. M. Küttel, L. Diederich, E. Schaller, O. Carnal, L. Schlapbach, Surf. Sci. 337 (1995) 812-818.

[41] L. Diederich, O. M. Küttel, P. Aebi, L. Schlapbach, Surf. Sci. 418 (1998) 219-239.

[42] P. John, M. D. Stoikou, Physical Chemistry 13 (2011) 11503-11510.

[43] G. Francz, P. Kania, G. Gantner, H. Stupp, P. Oelhafen, Phys. Status Solidi A 154 (1996) 91-108.

[44] J. Ristein, Surf. Sci. 600 (2006) 3677-3689.

[45] B. J. Waclawski, D. T. Pierce, N. Swanson, R. J. Celotta, J. Vac. Sci. Technol. 21 (1982) 368.

[46] R. Graupner, J. Ristein, L. Ley, Surf. Sci. 320 (1994) 201-207.

[47] Y. Qi, L. G. Hector, Phys. Rev. B 68 (2003) 1.

[48] S. Shin, M. Kaviany, T. Desai, R. Bonner, Phys. Rev. B 82 (2010) 1-4.

[49] K. P. Loh, X. N. Xie, S. W. Yang, J. S. Pan, P. Wu, Diam. Relat. Mater. 11 (2002) 1379-1384.

[50] G. Tas, J. J. Loomis, H. J. Maris, A. A. Bailes, L. E. Seiberling, Appl. Phys. Lett. 72 (1998) 2235-2237.

[51] W. A. Little, Canadian Journal of Physics 37 (1959) 334-349.

[52] E. T. Swartz, R. O. Pohl, Rev. Mod. Phys. 61 (1989) 605- 668.

[53] M. Kida, L. Weber, C. Monachon, A. Mortensen, J. Appl. Phys. 109 (2011) 1-9. 